\title{
Synbiotic meal decreases uremic toxins in hemodialysis individuals: A placebo-controlled trial
}

\author{
Rita de Cássia Stampini Oliveira Lopes ${ }^{\mathrm{a}, *}$, Jaqueline Maciel Vieira Theodoro ${ }^{\mathrm{a}}$, \\ Bárbara Pereira da Silva ${ }^{\mathrm{a}}$, Valéria Aparecida Vieira Queiroz ${ }^{\mathrm{b}}$, Maria Eliza de Castro Moreira ${ }^{\mathrm{a}}$, \\ Hilário Cuquetto Mantovani ${ }^{c}$, Helen Hermana Hermsdorff ${ }^{a}$, Hércia Stampini Duarte Martino ${ }^{a}$

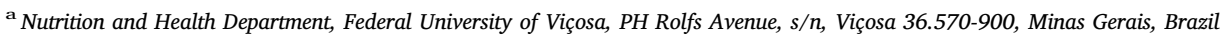 \\ b Embrapa Maize and Sorghum, Rodovia MG 424, Km 65, 35.701-970 Sete Lagoas, Minas Gerais, Brazil \\ ${ }^{\mathrm{c}}$ Microbiology Department, Federal University of Viçosa, PH Rolfs Avenue, s/n, Viçosa 36.570-900, Minas Gerais, Brazil
}

\section{A R T I C L E I N F O}

\section{Keywords:}

Bifidobacterium longum

Chronic kidney disease

Indoxyl sulfate

p-Cresylsulfate

Sorghum bicolor L.

Urea

\begin{abstract}
A B S T R A C T
Generation of uremic toxins p-cresylsulfate (p-CS), indoxyl sulfate (IS) and indole 3-acetic acid (IAA) in hemodialysis (HD) individuals may be associated with the gut flora and recognized markers of disease progression. This study investigated the effect of synbiotic meal on uremic toxins in HD individuals. We conducted randomized singleblind and placebo-controlled intervention study with $58 \mathrm{HD}$ subjects (20F/38M, 63.1 \pm 10.9 -old) who were randomly allocated in synbiotic group (SG, $40 \mathrm{~g}$ of extruded sorghum plus $100 \mathrm{~mL}$ of unfermented probiotic milk) or control group (CG, $40 \mathrm{~g}$ of extruded corn plus $100 \mathrm{~mL}$ of pasteurized milk), during 7-wk Metabolic markers and uremic toxins, fecal concentration of short chain fatty acid and $\mathrm{pH}$ value was determined. The SG group had decreased serum p-CS and IS, as well as decreased urea concentration $(p<.05)$ compared to CG. SG showed higher fecal butyric acid and lower pH compared to baseline and SC (p < .05). In addition, serum p-CS and fecal pH were positively correlated to urea concentration in SG participants at the endpoint. The consumption of the synbiotic meal during 7-wk reduced colonic $\mathrm{pH}$, and reduced serum uremic (p-CS and IS) toxins and urea in HD subjects.
\end{abstract}

\section{Introduction}

Chronic kidney disease (CKD) is a worldwide public health problem with estimated prevalence between $10 \%$ to $13 \%$ (Hill et al., 2016). Cardiometabolic risk factors are among several complications of CKD and hemodialysis (HD) treatment and cardiovascular disease is the major cause of morbidity and mortality in HD patients (Bansal et al., 2016). Atherosclerosis pathogenesis in CKD patients has been associated with traditional risk factors, such as hypertension, diabetes, and age, as well as non-traditional risk factors, such as uremic toxins and anemia (Ito \& Yoshida, 2014).

CKD patients have been presenting progressive retention of uremic toxins, which can negatively impact many body functions and increase cardiovascular mortality (Borges et al., 2017; Vaziri, 2016). Uremic toxins, p-cresylsulfate (p-CS), indoxyl sulfate (IS) and indole 3-acetic acid (IAA) are toxins commonly used as markers of CKD progression
(Fukuuchi et al., 2002), because these metabolites accumulate in the blood flow and may contribute to uremic syndrome. They induce renal tubular cells to produce free radical, promote vascular calcification, and aortic wall thickening (Evenepoel, Meijers, Bammens, \& Verbeke, 2009).

Reducing proteolytic bacteria are known to regulate uremic toxin synthesis though probiotics and/or prebiotics use (Evenepoel et al., 2009; Rossi, Kerenaftali, Johnson, \& Campbell, 2012); however, this mechanism has been little explored. Probiotic is a live microbial food supplement that beneficially affects the host animal by improving its gut microbiota balance when administered in adequate quantities (Ashraf \& Shah, 2011; Park \& Bae, 2015). According to legislation, the minimum recommendation of viable cells for a product to be considered probiotic is of $10^{8} \mathrm{CFU} / 100 \mathrm{~mL}$ (Anvisa, 2017) Studies have highlighted the use of probiotics with Bifidobacterium longum for metabolic control in CKD patients (Borges et al., 2017; Lopes et al., 2018;

\footnotetext{
Abbreviations: CKD, chronic kidney disease; HD, hemodialysis; p-CS, p-cresylsulfate; IS, indoxil sulfate; IAA, indole 3-acetic acid; CG, control group; SG, synbiotic

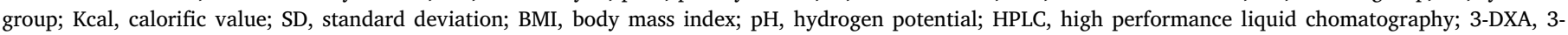
deoxyanthiocyanidins

* Corresponding author.

E-mail address: rita.lopes@ufv.br (R.d.C.S.O. Lopes).
} 
Ogawa et al., 2012; Takayama, Taki, \& Niwa, 2003).

Sorghum (Sorghum bicolor L.) is a cereal from the Poaceae family native to the tropical regions of Africa. This cereal has a nutritional composition similar to corn and is a source of dietary fibers and bioactive compounds, predominantly in the grain pericarp, which can help control of organic residues in the body (Cardoso, Pinheiro, Martino, \& Pinheiro-Sant'Ana, 2017; Lopes et al., 2018; VázquezAraújo, Chambers, \& Cherdchu, 2012). Cultivars with pigmented pericarp, such BRS 305, present high levels of condensed tannins, called proanthocyanidins, which have high antioxidant activity and can attenuate uremia, cardiovascular dysfunction and mortality in CKD patients (Cardoso et al., 2017; Lopes et al., 2018; Moraes et al., 2015). However, no studies were found assessing the functionality of sorghum on uremic toxins in CKD patients.

Thus, in this study we hypothesized that consumption of synbiotic meal (synbiotic group - SG) is better than placebo meal (control group $\mathrm{CG}$ ) to reduce colonic $\mathrm{pH}$, increase short chain fatty acid production by gut microbiota, and subsequently reduce serum uremic toxins and metabolic markers in HD subjects. Our aim was to investigate the effect of the synbiotic meal (extruded sorghum breakfast meal plus unfermented probiotic dairy beverage) on uremic toxins in HD individuals during a 7-wk intervention.

\section{Material and methods}

\subsection{Subjects}

One hundred and twelve subjects in HD attended in a single dialysis center (Brazil) were evaluated for eligibility. Of these, one hundred and seven met the inclusion criteria and were selected for the study. The inclusion criteria and exclusion criteria were based accordingly to Lopes et al. (2018). We assessed the dialysis prescription and included the following items in dialysis duration assessment: $4 \mathrm{~h}$ per session, 3 times per week, with a blood flow ranging from 200 to $250 \mathrm{~mL} / \mathrm{min}$ and dialysate flow of $500 \mathrm{~mL} / \mathrm{min}$.

Thus, ninety-nine selected participants were randomly distributed into two groups, placebo (control) or synbiotic group. At the end of the intervention, 58 volunteers completed the study (Fig. 1).

Sample size was calculated considering: (1) difference in the concentration of IS as main outcome (Takayama et al., 2003); (2) published SD ( \pm 15.5 ) for IS concentration; (3) statistical power of $90 \%$; and (4) significance level of $5 \%$. For all these criteria, the sample size required was a minimum of 22 volunteers per group (Dean, Sullivan, \& Soe, 2014.).

The study protocol was approved by the Human Research Ethics Committee of the Universidade Federal de Viçosa (protocol number 701.796/2014) and was registered at www.ensaiosclinicos.gov.br, with ID number RBR-2d9ny6. All participants read and signed the written informed consent.

\subsection{Study design}

This is a controlled, randomized, simple blind study, during 7 weeks, respecting the blood collection routine of the nephrology sector. Socio-demographic and clinical data were obtained before the intervention period by medical records and interviews. Anthropometric data were measured. The participants were randomly assigned to one of the groups: synbiotic group - SG $(100 \mathrm{~mL}$ of probiotic dairy drink and $40 \mathrm{~g}$ of extruded sorghum flakes) or control group -CG $(100 \mathrm{~mL}$ of pasteurized milk and $40 \mathrm{~g}$ of extruded corn flakes) (Fig. 2). The distribution of food occurred as described previously by Lopes et al. (2018).

At the beginning and at the end of the intervention, blood samples were drawn before the hemodialysis session by a qualified professional. After, blood samples were centrifuged and immediately stored at $-80^{\circ} \mathrm{C}$. Stool samples were collected by participants in sterile bottles, kept at snap frozen $\left(-18^{\circ}\right)$ until the time of hemodialysis. Participants used styrofoam packaging with ice cubes to transport samples to the nephrology sector. Samples were collected during HD sessions, aliquoted and stored at $-80^{\circ} \mathrm{C}$.

\subsection{Products preparation}

The grains were grown in the experimental field of Embrapa Milho e Sorgo, located in Sete Lagoas, Brazil. The BR 305 sorghum, hybrid with brown pericarp with tannins, was cultivated from April to July 2014 and corn grains were cultivated in the $2013 / 2014$ crop. After the harvest, the grains were packed in plastic bags and sent to Embrapa Agroindústria de Alimentos, Rio de Janeiro, Brazil for processing.

The whole grains, previously milled and added of $10 \%$ refined sugar and $0.5 \% \mathrm{NaCl}$, were processed in a co-rotating twin-screw (Model Evolum HT25,Clextral Inc., Firminy, France) as described in Lopes et al. (2018)The extrudates were oven dried at $60^{\circ} \mathrm{C}$ for $2 \mathrm{~h}$, packed in polyethylene bags and transported to the Universidade Federal de Viçosa, where they were sampled ( $40 \mathrm{~g}$; proportion stipulated according to the percentage of food fiber present in extruded sorghum) in plastic packaging, sealed by means of a sealing machine, labeled and stored at $5{ }^{\circ} \mathrm{C} \pm 2{ }^{\circ} \mathrm{C}$.

The dairy drinks used in the study were pasteurized milk, used in the control group (CG) and pasteurized milk with addition of the probiotic bacterium Bifidobacterium longum BL-G301 (Granotec do Brasil S.A.), used in the synbiotic group (SG). The production of the probiotic dairy beverage was carried out weekly in the Universidade Federal de Viçosa, according to described by Oliveira et al. (2017). The drinks were stored under refrigeration at $4{ }^{\circ} \mathrm{C} \pm 2{ }^{\circ} \mathrm{C}$ for up to seven days. The viability of the probiotic product was performed weekly and the concentration of viable Bifidobacterium longum BL-G301 cells averaged $7.4 \times 10^{8} \pm 5.4 \times 10^{8} \mathrm{CFU} / 100 \mathrm{~mL}$ portion, with the minimum concentration being $2.5 \times 10^{8}$ and the maximum of $1.5 \times 10^{9} \mathrm{CFU} /$ $100 \mathrm{~mL}$. The chemical composition of the products used in the study is described in Table 1 (Lopes et al., 2018).

\subsection{Data collection}

All anthropometric measurements were performed at the end of the hemodialysis session, after $30 \mathrm{~min}$ of hemodynamic balance. The anthropometric measures included in the study were weight $(\mathrm{kg})$ and height $(\mathrm{cm})$, which were performed according to previously standardized procedures (Jellife, 1968; Lipschitz, 1994; WHO, 1997). The body mass index (BMI) was calculated by ratio between weight and height, and the weigth-status of the individuals was calculated according to the cut-off points of the World Health Organization for adults (WHO, 1997) and Lipschitz for elderly (Lipschitz, 1994).

Food consumption was evaluated by the three-day food recall, considering a weekend day, one day without hemodialysis and one referring to the day of treatment (Bingham, Welch, \& Cassidy, 1994).The dietary recall was evaluated in Dietpro ${ }^{\circledR}$ nutrition software (version 20.0). The information of the recall was transferred to the software by two trained typists and data entered by two other individuals.

The metabolic markers analyzed were obtained from the medical record and refer to the period before the intervention and those collected after the intervention: albumin, urea, creatinine, potassium, phosphorus, calcium, hemoglobin, hematocrit, iron and alkaline phosphatase.

The uremic toxins (IS, p-CS, and IAA) were quantified, in triplicate, by high-performance liquid chromatography (HPLC) with fluorescent detection. Briefly, plasma samples were processed as described in Meert et al. (2012). This was diluted with water and heated $\left(95^{\circ} \mathrm{C}, 30 \mathrm{~min}\right)$. After $10 \mathrm{~min}$ in ice, samples were centrifuged $(13,000 \mathrm{rpm}$ in bench centrifuge, $4^{\circ} \mathrm{C}, 20 \mathrm{~min}$ ) and the supernatant was ultra-fltered with a 30-kDa cutof membrane (Amicon Ultra, Millipore). The ultrafiltered 


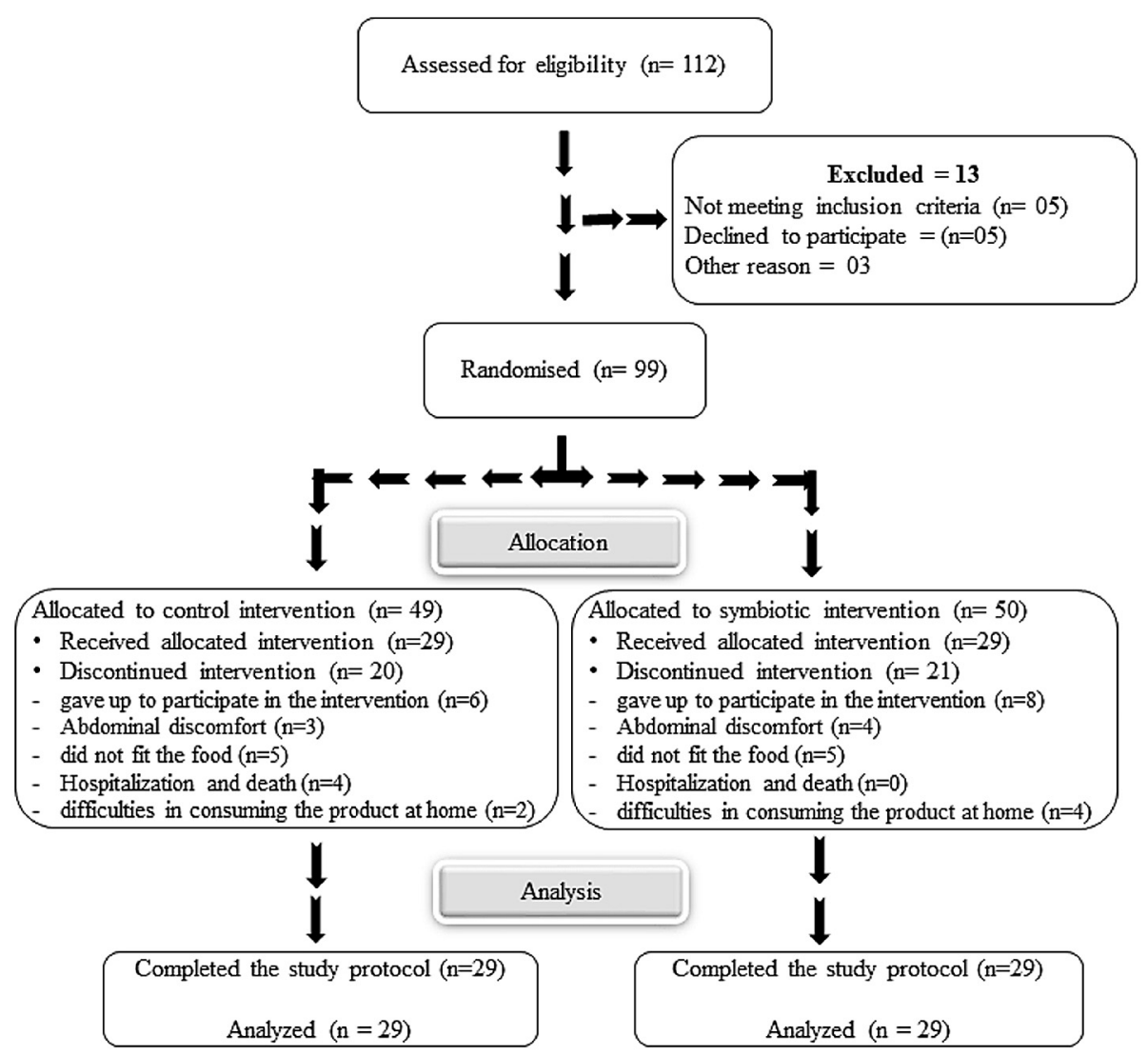

Fig. 1. CONSORT diagram showing the flow of participants through each stage of the trial. CONSORT Consolidated Standards of Reporting Trials.

serum $(10 \mu \mathrm{L})$ was injected into an HPLC system (Shimadzu Prominence) consisting of a Rheodyne injector (model 7125), a quaternary pump (Shimadzu LC-20 CE), controlled by the LC Solution software, and a fluorescence detector (Shimadzu RF-20A). The calibrators were prepared in a pooled healthy plasma matrix and processed exactly as the plasma samples. The amounts added to the $100 \mu \mathrm{L}$ matrix (IS $0.2-28 \mu \mathrm{mol}$; p-CS $0.63-79.6 \mu \mathrm{mol}$; IAA $0.02-3 \mu \mathrm{mol}$ ) were chosen to match plasma concentrations ranging from the normal to uremic concentrations of the three toxins. The inferior limits of quantification (LOQ) of the toxins were determined as $10 \times \sigma / \mathrm{S}$, where $\sigma$ is the standard deviation of the response (calibrators at the lowest concentrations) and $\mathrm{S}$ is the slope of the linear regression. Plasma samples $(100 \mu \mathrm{L})$ were spiked with the three authentic standards in the normal or uremic ranges: IS (1.1 or $24 \mu \mathrm{mol}), \mathrm{p}-\mathrm{CS}(2.6$ or $42.5 \mu \mathrm{mol})$ and IAA ( 0.11 or $2 \mu \mathrm{mol}$ ), and processed as described above (Stockler-Pinto et al., 2018). Separation was achieved with C8 Luna column
(Phenomenex, Luna $5 \mu \mathrm{m}, 100 \AA$, $150 \times 4,6 \mathrm{~mm}$ ), eluted with $50 \mathrm{mM}$ ammonium formate, $\mathrm{pH} 3.0$ and methanol, whose proportion increased from $35 \%$ to $70 \%$ along the run, at a flow rate of $0.7 \mathrm{~mL} / \mathrm{min}$. During the run, the fluorescence wavelengths varied $\lambda \operatorname{exc}=280 \mathrm{~nm}$ and $\lambda$ emi $=383 \mathrm{~nm}$ to IS and $\lambda$ exc $=265 \mathrm{~nm}$ and $\lambda$ emi $=290 \mathrm{~nm}$ to $\mathrm{p}$-CS and IAA.

The fecal hydrogen potential $(\mathrm{pH})$ was measured in digital $\mathrm{pHmeter}$ T-1000 (Tekna, São Paulo, Brazil). One gram of feces was filled in falcon-type tube ( $15 \mathrm{~mL}$ capacity) and $10 \mathrm{~mL}$ of ultrapure water was added. After vortex homogenization, the $\mathrm{pH}$ was read.

For the extraction of organic acids in fecal contents, around $500 \mathrm{mg}$ of feces were homogenized with the addition of $1 \mathrm{~mL}$ of ultrapure water. The samples were centrifuged (Himac CT 15RE, Hitachi) at $12000 \mathrm{~g}$ for $10 \mathrm{~min}$ at $4^{\circ} \mathrm{C}$. Then, supernatants were treated as described by Siegfried, Ruckemann, and Stumpf (1984). Organic acids (acetic, propionic and butyric acid) were determined by high performance

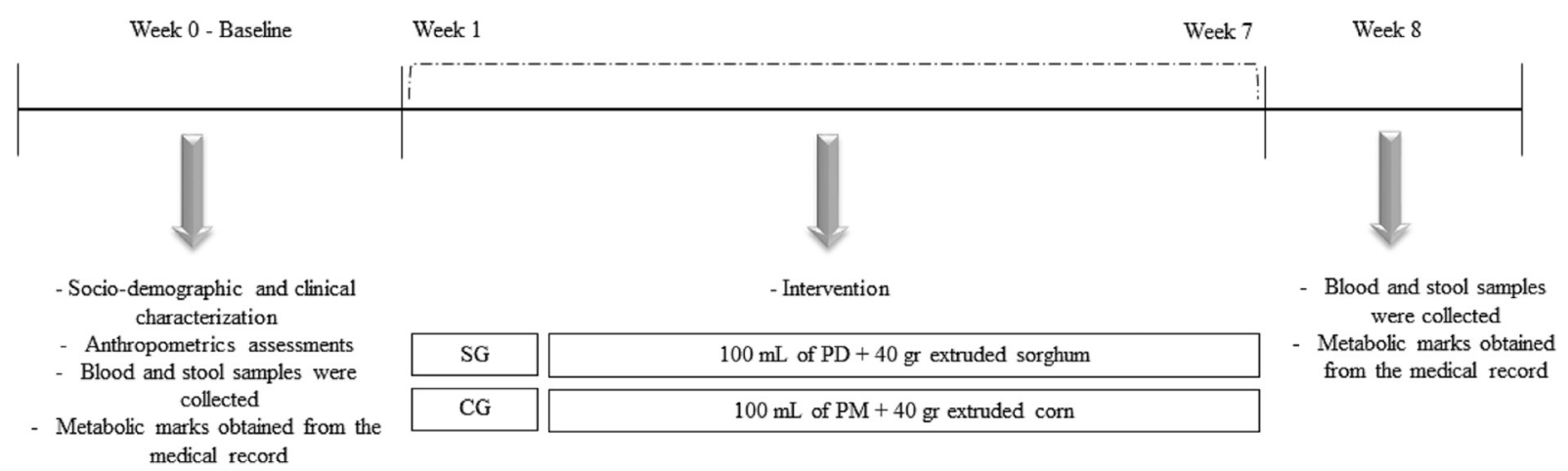

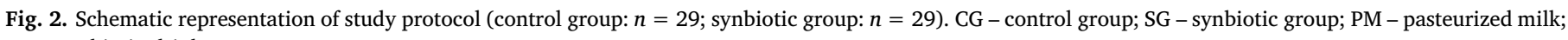
$\mathrm{PD}$ - probiotic drink. 
Table 1

Chemical composition of the products used during the intervention; unit per serving. ${ }^{\text {a }}$

\begin{tabular}{llll}
\hline & Synbiotic product & Control product & $\mathrm{p}$ \\
\hline Energy (Kcal) & $196.16 \pm 0.67$ & $200.25 \pm 2.06$ & 0.031 \\
Carbohydrate (g) & $33.42 \pm 0.29$ & $33.52 \pm 0.15$ & 0.617 \\
Protein (g) & $7.90 \pm 0.42$ & $8.56 \pm 0.33$ & 0.140 \\
Lipids (g) & $3.36 \pm 0.05$ & $3.53 \pm 0.04$ & 0.012 \\
Total Fiber (g) & $3.54 \pm 0.45$ & $2.91 \pm 0.35$ & 0.037 \\
Phosphorus (mg) & $136.00 \pm 0.00$ & $143.20 \pm 0.40$ & $<0.001$ \\
Potassium (mg) & $141.20 \pm 1.20$ & $140.80 \pm 0.40$ & 0.613 \\
Iron (mg) & $2.23 \pm 0.10$ & $1.45 \pm 0.01$ & $<0.001$ \\
Calcium (mg) & $160.80 \pm 0.40$ & $140.93 \pm 1.01$ & $<0.001$ \\
Tannin (mg catechin eq.) & $28.40 \pm 0.07$ & - & \\
Phenolic compounds (mg gallic & $44.20 \pm 0,84$ & $32.60 \pm 0.36$ & $<0.001$ \\
$\quad$ ac. eq.) & & & \\
Total 3 - DXAs (mg) & $72.78 \pm 12.92$ & - & \\
\hline
\end{tabular}

a Calculation based on the chemical composition of the extruded and the chemical composition of pasteurized milk. Portion: $40 \mathrm{~g}$ of extruded cereal and $100 \mathrm{~mL}$ milk drink.

liquid chromatography (HPLC) in a Dionex Ultimate 3000 Dual detector HPLC (Dionex Corporation, Sunnyvale, CA, USA) coupled to a refractive index (RI) Shodex RI-101.

The organic acids were separated on a Phenomenex Rezex ROA ion exclusion column $(300 \times 7.8 \mathrm{~mm})$ (Phenomenex Inc. Torrance, CA, USA) maintained at $45^{\circ} \mathrm{C}$. Analyses were performed isocratically in duplicate samples under the following conditions: mobile phase $\mathrm{H}_{2} \mathrm{SO}_{4}$ $5 \mathrm{mmoll}^{-1}$, flow rate $0.7 \mathrm{~mL} \mathrm{~min}^{-1}$, column temperature $40^{\circ} \mathrm{C}$, injection volume $20 \mu \mathrm{L}$. Five calibration standards were prepared by diluting the stock solutions 2, 4, 8 and 16 times in the mobile phase. The stock solution contained the following concentrations $\left(\mathrm{mmoll}^{-1}\right)$ of pure acids: acetic 20, propionic 10 and butyric acid 10. The correlation coefficients of the external standards were 0.999, 0.999 and 0.998 for acetic, propionic and butyric acids, respectively. Quantitation limits were calculated based on the standard deviation of the response and the slope of the calibration curves. The following LOD and LOQ values were obtained $\left(\mathrm{mmoll}^{-1}\right): \quad \operatorname{LOD}_{\text {Acetic }}=0,023$ and $\mathrm{LOQ}_{\text {Acetic }}=0,069 ; \quad \mathrm{LOD}_{\text {Propionic }}=0,232$ and $\mathrm{LOQ}_{\text {Propionic }}=0,077$; $\mathrm{LOD}_{\text {Butyric }}=0,013$ and $\mathrm{LOQ}_{\text {Butyric }}=0,038$.

\subsection{Statistical analysis}

The recorded data were reviewed in order to detect missing information and inconsistencies. Subsequently a descriptive analysis of the general characteristics of the participants per group was performed. Quantitative variables with normal distribution (according to graphical analysis, asymmetry and kurtosis coefficients and Shapiro-Wilk test) were expressed as mean and standard deviation (SD) and those that did not present normal distribution were expressed in median and p25-p75. Differences between groups were assessed by the chi-square test (categorical variables), Student's $t$-test or Mann-Whitney test (numerical variables). Paired-t-test or the Wilcoxon test was used to assess differences within the groups. Spearman's rank-correlation coefficient was used to assess the association of serum urea with p-CS and fecal $\mathrm{pH}$, for each tested group. Statistical analysis was performed using the Statistical Package for Social Sciences 20.0 program (SPSS, Inc.,

Table 2

Baseline characteristics of HD subjects, according to intervention groups.

\begin{tabular}{|c|c|c|c|c|}
\hline Variables & All patients $(n=58)$ & Control group $(n=29)$ & Synbiotic group $(n=29)$ & $P$ value \\
\hline Age (year) ${ }^{\mathrm{a}}$ & $63.10 \pm 10.90$ & $63.03 \pm 10.77$ & $63.17 \pm 11.16$ & 0.962 \\
\hline BMI $\left(\mathrm{Kg} \cdot \mathrm{m}^{-2}\right)^{\mathrm{a}}$ & $23.99 \pm 3.65$ & $23.26 \pm 2.87$ & $24.74 \pm 4.24$ & 0.151 \\
\hline $\operatorname{Sex}^{\mathrm{c}}$ & & & & 0.269 \\
\hline Male & $38(65.52)$ & $21(72.4)$ & $17(58.6)$ & \\
\hline Female & $20(34.48)$ & $8(27.6)$ & $12(41.4)$ & \\
\hline HD time (months) ${ }^{\mathrm{b}}$ & $32(14.00 ; 77.00)$ & $31(14.00 ; 71.50)$ & $34(15.50 ; 34.00)$ & 0.994 \\
\hline Cause of Kidney Disease ${ }^{c}$ & & & & 0.973 \\
\hline Diabetes & $26(44.8)$ & $13(44.8)$ & $13(44.8)$ & \\
\hline Hypertensive nephrosclerosis & $13(22.4)$ & $6(20.7)$ & $7(24.3)$ & \\
\hline Glomerulonephritis & $3(5.1)$ & $2(7.0)$ & $1(3.4)$ & \\
\hline Polycystic kidney disease & $2(3.5)$ & $1(3.4)$ & $1(3.4)$ & \\
\hline Obstructive uropathy & $2(3.5)$ & $1(3.4)$ & $1(3.4)$ & \\
\hline Other causes & $12(20.7)$ & $6(20.7)$ & $6(20.7)$ & \\
\hline \multicolumn{5}{|l|}{ Metabolic markers $^{\mathrm{a}}$} \\
\hline Creatinine (mg/dL) & $8.6 \pm 3.07$ & $8.71 \pm 3.32$ & $8.48 \pm 2.84$ & 0.78 \\
\hline Potassium (mEq/l) & $5.61 \pm 0.86$ & $5.71 \pm 0.96$ & $5.51 \pm 0.75$ & 0.37 \\
\hline Urea $(\mathrm{mg} / \mathrm{dL})$ & $148.03 \pm 20.54$ & $143.13 \pm 19.53$ & $152.93 \pm 20.67$ & 0.07 \\
\hline Iron $(\mu \mathrm{g} / \mathrm{dL})$ & $58.50 \pm 22.33$ & $56.46 \pm 25.45$ & $62.03 \pm 17.46$ & 0.49 \\
\hline Hematocrit $(\%)$ & $33.62 \pm 8.90$ & $33.25 \pm 5.99$ & $34.26 \pm 11.29$ & 0.91 \\
\hline Red blood cell (millions $/ \mathrm{mm}^{3}$ ) & $4.00(3.00 ; 4.00)$ & $4.00(3.00 ; 4.00)$ & $4,00(3.00 ; 4.00)$ & 1.00 \\
\hline Hemoglobin $(\mathrm{g} / \mathrm{dL})$ & $11.05(9.57 ; 12.55)$ & $11.40(9.80 ; 12.80)$ & $11.00(9.45 ; 12.15)$ & 0.59 \\
\hline Alkaline Phosphatase (U/I) & $320.50(251.50 ; 495.00)$ & $322.00(226.00 ; 520.00)$ & $319.00(256.00 ; 485.00)$ & 0.82 \\
\hline Phosphrus (mg/dL) & $5.50(4.80 ; 6.50)$ & $5.80(3.13 ; 8.90)$ & $5.50(3.60 ; 7.30)$ & 0.98 \\
\hline Albumin $(\mathrm{g} / \mathrm{dL})$ & $4.00(3.66 ; 4.10)$ & $4.00(3.60 ; 4.10)$ & $4.00(3.71 ; 4.09)$ & 0.75 \\
\hline \multicolumn{5}{|l|}{ Uremic Toxins $(\mu \mathrm{M})$} \\
\hline $\mathrm{IAA}^{\mathrm{a}}$ & $17.20(12.82 ; 26.95)$ & $15.41(3.53 ; 62.28)$ & $20.34(5.12 ; 56.86)$ & 0.09 \\
\hline $\mathrm{IS}^{\mathrm{b}}$ & $160.59 \pm 75.72$ & $167.87 \pm 75.88$ & $153.30 \pm 76.20$ & 0.47 \\
\hline $\mathrm{p}-\mathrm{CS}^{\mathrm{b}}$ & $306.01(240.17 ; 584.64)$ & $288.70(248.66 ; 578.71)$ & $339.06(213.27 ; 593.16)$ & 0.99 \\
\hline \multicolumn{5}{|l|}{ Organic acid (mmol/L) } \\
\hline Acetic acid & $4.33 \pm 1.98$ & $4.92 \pm 2.15$ & $3.79 \pm 1.69$ & 0.07 \\
\hline Propionic acid & $2.69 \pm 2.44$ & $3.04 \pm 2.84$ & $2.39 \pm 2.07$ & 0.39 \\
\hline Butyric acid & $2.27 \pm 1.72$ & $2.44 \pm 1.88$ & $2.25 \pm 1.62$ & 0.91 \\
\hline
\end{tabular}

Level of significance 5\%. BMI - body mass index; IAA - indole 3-acetic acid, IS - indoxyl sulfate, p-CS - p-cresyl sulfate.

a Mean \pm Std. Deviation, Independent Samples $t$-test.

b median (p25; p75), Mann-Whitney test.

c n (\%), Chi-Square Tests. 
Chicago, IL, USA) and the significance level ( $\alpha$ ) was considered equal to $5 \%$.

\section{Results}

\subsection{Baseline characteristics of the participants}

58 participants showed hemodialysis time ranged from 3 to 245 months and $63.1 \pm 10.9$ years old. The patients presented diabetes mellitus (44.8\%) and hypertensive nephrosclerosis (22.4\%) as the main diseases related to CKD. At the baseline, $19.6 \%$ of HD patients presented underweight, $52.9 \%$ were normal-weight and $27.4 \%$ were overweight/obese. The randomization of the groups was adequate, since they did not present differences in age, BMI, sex, presence of diabetes, HD time, metabolic markers, uremic toxins and SCFA $(p>$.05) (Table 2). The synbiotic and the control groups presented no differences in macronutrients and dietary fiber consumption during the intervention $(\mathrm{p}>.05)$.

\subsection{BMI and metabolic markers}

BMI values showed no statistical difference in the SG $(24.94 \pm 4.31)$ in relation to the CG $(23.84 \pm 2.75)(p>.05)$. The HD patients who intake the synbiotic meal and CG presented similar ( $p>.05)$ nutritional state classification, respectively, underweight (16.0\% and $15.4 \%)$, normal-weight $(52.0 \%$ and $65.4 \%)$ and overweight/obese (32.0\% and $19.2 \%)$.

The SG decreased urea concentration and showed a higher percentage $(82.8 \% ; n=24)$ of adequation pf serum albumin $(p<.05)$ compared to CG. The other metabolic markers showed no significant difference between the groups $(p>.05)$. The CG and SG groups decreased serum alkaline phosphatase concentration and an increased in an albumin in relation to the baseline $(p<.05)$. However, only SG decreased urea and phosphorus concentration in relation to baseline $(\mathrm{p}<.05)$ (Fig. 3).

\subsection{Organic acids, fecal $p H$, uremic toxins and their correlations}

The SG presented higher concentration of acetic, butyric, propionic acids at endpoint $(7-w k)$, compared to baseline $(p<.05)$ and CG presented higher concentration of acetic and propionic acid $(p<0,05)$ at endpoint (7-wk), compared to baseline ( $\mathrm{p}<.05)$, with no difference between groups. In addition to, SG presented lower fecal pH than CG, after 7-wk $(p<.05)$ (Table 3).

The SG presented decreased serum $\mathrm{p}-\mathrm{CS}$ and IS concentration ( $p<.05)$ compared to the CG. In addition, the CG had decreased $(\mathrm{p}<.05)$ serum $\mathrm{p}$-CS concentration while the SG had decreased IAA, IS and p-CS concentration at endpoint (7-wk) compared to baseline (Fig. 4). In relation to delta values (delta final - delta initial), the SG presented a larger decrease in serum IS and p-CS concentration $(\mathrm{p}<.05)$ than the CG (Fig. 4). Delta values for organic acids had no distinction $(p>.05)$.

Analysis between delta fecal $\mathrm{pH}$ and delta solute concentrations presented no differences. However, urea and p-CS correlation and serum urea and fecal $\mathrm{pH}$ correlation was positive in SG at the endpoint (Fig. 5A-B). The control group did not present differences $(p>.05)$ between these variables.

\section{Discussion}

This research is the first to investigate the effects of synbiotic meal (extruded sorghum breakfast meal plus unfermented probiotic dairy beverage) on metabolic and uremic toxins control in HD individuals.

The main result of this study was that synbiotic meal decreased serum uremic toxins in HD subject after 7-wk of intervention. This result demonstrated protection effect, since CKD patients present a progressive retention of uremic toxins, with a negative impact on many body functions and increase in cardiovascular mortality. The increase of the toxins in the bloodstream can increase intestinal permeability caused by influx of urea and expansion of urease-possessing bacteria (Borges et al., 2017; Vaziri, 2016). Probably, synbiotic meal

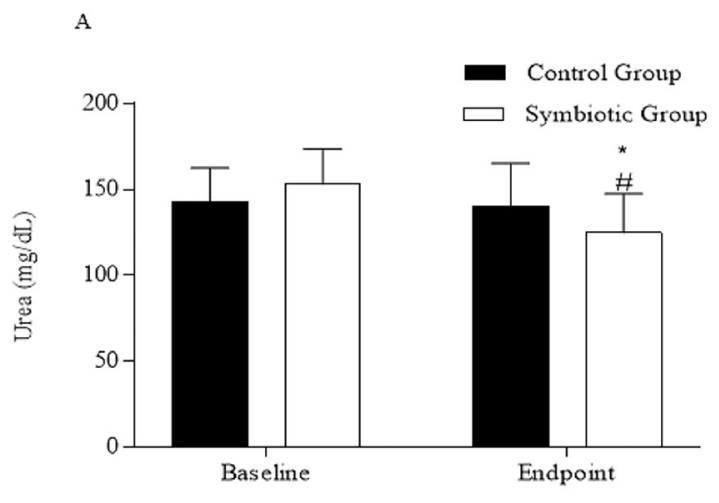

B

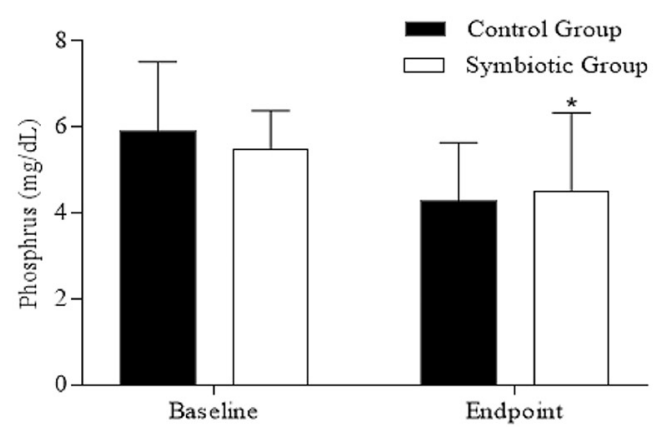

$\mathrm{C}$

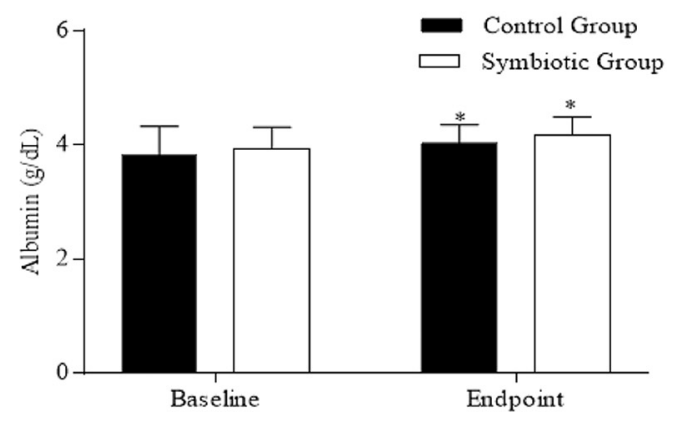

Fig. 3. Metabolic markers of HD individuals before and after 7-wk. A- Urea; B- Phosphorus; C- Albumin. * $p<.05$ from paired-t-test for comparison between baseline and endpoint values for synbiotic group; $\# \mathrm{p}<.05$ from Student-t-test for comparison between groups (control vs. synbiotic) at the endpoint values. 
Table 3

Organic acid concentration and fecal pH in subjects with chronic kidney disease undergoing hemodialysis.

\begin{tabular}{|c|c|c|c|c|c|c|c|}
\hline \multirow[t]{2}{*}{ Variables } & \multicolumn{2}{|l|}{ Control group } & \multicolumn{3}{|c|}{ Synbiotic group } & \multirow[b]{2}{*}{$\mathrm{P}^{\mathrm{a}}$} & \multirow[b]{2}{*}{$\mathrm{P}^{\mathrm{b}}$} \\
\hline & Baseline & Endpoint & $\mathrm{P}^{\mathrm{a}}$ & Baseline & Endpoint & & \\
\hline \multicolumn{8}{|c|}{ Organic acids $\left(\mathrm{mmol}^{-1}\right)$} \\
\hline Acetic acid & $4.92 \pm 2,15$ & $7.90 \pm 3.80$ & $0.02 *$ & $3.79 \pm 1.69$ & $6.51 \pm 2.24$ & $0.006^{*}$ & 0.08 \\
\hline Propionic acid & $3.04 \pm 2,84$ & $6.17 \pm 3.66$ & $0.02 *$ & $2.39 \pm 2.07$ & $6.36 \pm 3.70$ & $0.002^{*}$ & 0.93 \\
\hline Butyric acid & $2.44 \pm 1,88$ & $3.64 \pm 2.94$ & 0.18 & $2.25 \pm 1.62$ & $3.91 \pm 2.41$ & $0.004 *$ & 0.69 \\
\hline Fecal pH & $6.16 \pm 1.25$ & $5.75 \pm 1.03$ & $0.03^{*}$ & $6.39 \pm 0.99$ & $5.12 \pm 0.90$ & $0.000^{*}$ & $0,04 *$ \\
\hline
\end{tabular}

Mean \pm Std. Deviation, Independent Samples $t$-test or paired-t-test; Level of significance $5 \%$.

Median (p25; p75) Mann-Whitney or Wilcoxon test; Level of significance 5\%.

a Statistical intragroup analysis (baseline vs. endpoint).

b Statistical analysis between groups at endpoint.
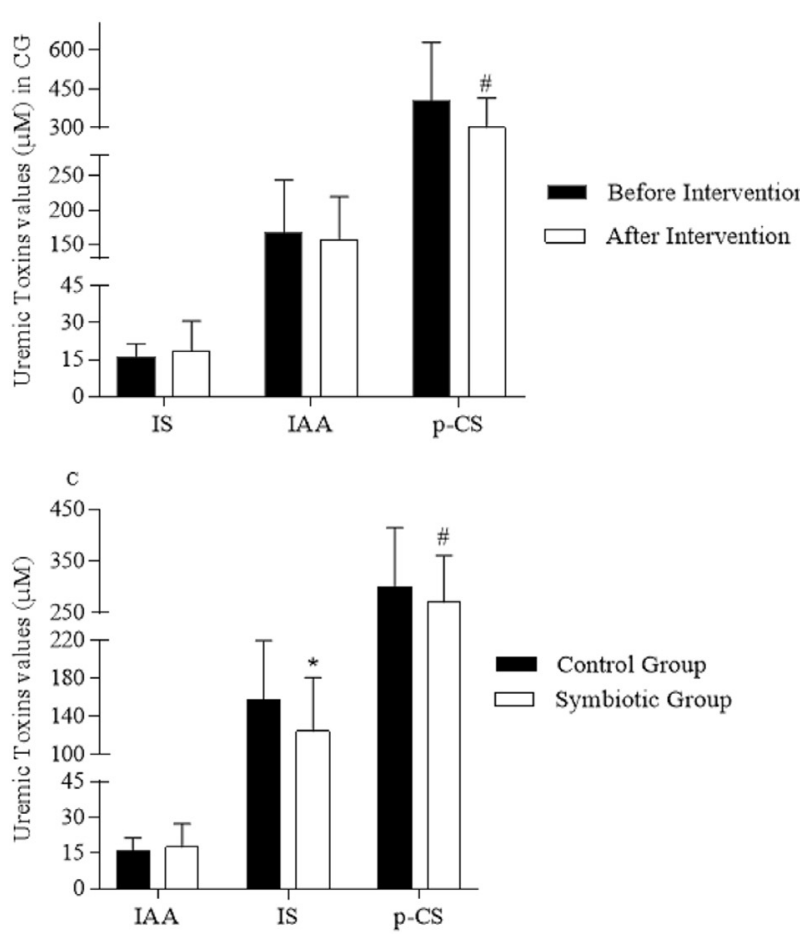

B
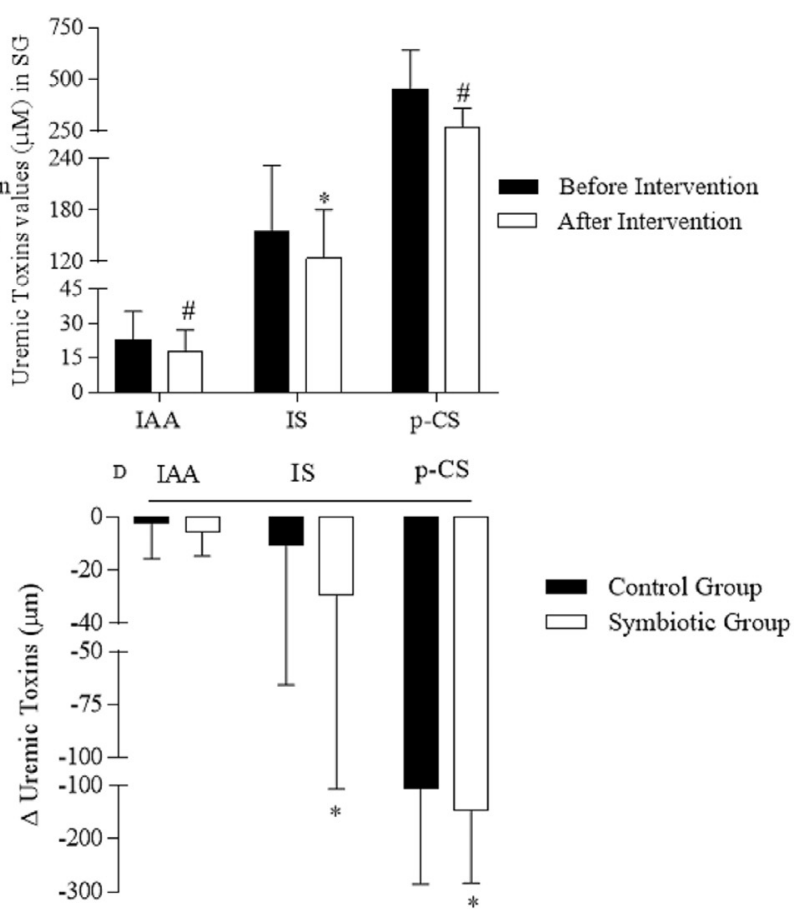

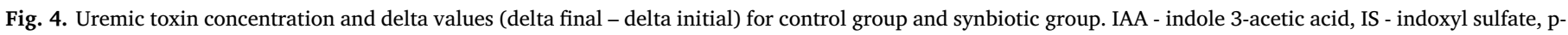

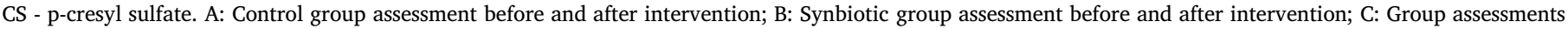
after intervention; D: Group delta values.

consumption may reduce $E$. Coli and putrefactive bacteria in the intestine, which reduces intestinal permeability and uremic toxins production and diffusion from the intestinal lumen into the bloodstream (Skrypnik \& Suliburska, 2017). These effect may decrease a renal fibrosis, delay the progression of CKD, and improve azotemia (Vaziri, 2016). Our results differ of some studies with synbiotic therapy in CKD patients (Nakabayashi et al., 2011; Rossi et al., 2016) because it presented a reduction in the serum concentrations of both toxins, IS and pCS.

The second point is that SG group decreased serum urea after 7-wk of intervention. The synbiotic meal may decrease production of urea from ammonia in the liver by decreasing ammonia production by urease and its influx into the gastrointestinal tract. In addition, SG may decrease ammonium hydroxide production from ammonia, which decrease the gut's luminal $\mathrm{pH}$, preventing dysbiosis and intestinal mucosa alterations and, subsequently, decreasing uremic toxins production and systemic inflammation (Dehghani, Heidari, Mozaffari-Khosravi, NouriMajelan, \& Dehghani, 2016; Vaziri, 2016; Wong et al., 2014). The concentration of dietary fibers (3,6 $\mathrm{g}$ /portion) and bioactive compounds (condensed tannins: $13.06 \pm 1.52 \mathrm{mg}$ catechin eq/portion; flavonoids: $76.78 \pm 12.92 \mu \mathrm{g}$ total $3 \mathrm{DXAs} /$ portion; phenolic compounds: $44.20 \mathrm{mg}$ of gallic acid eq/portion) in extruded sorghum and Bifidobacterium longum $\left(7.4 \times 10^{8} \pm 5.4 \times 10^{8} \mathrm{CFU} / 100 \mathrm{~mL}\right.$ portion) in the probiotic milk (Lopes et al., 2018) can decrease ammonium hydroxide production from ammonia, which decrease the gut's luminal $\mathrm{pH}$, preventing dysbiosis and intestinal mucosa alterations and, subsequently, decreasing uremic toxins production and systemic inflammation (Dehghani et al., 2016; Vaziri, Zhao, \& Pahl, 2016; Wong et al., 2014). The polymeric tannins from sorghum can interact with starch production resistant starch. Then, the dietary fiber fermented by intestinal microbiota can acidify the colonic environment, which inhibit the growth of bacteria acid, such as species producers of urease enzyme (Cardoso et al., 2017; Dehghani et al., 2016; Vaziri, 2016). Similar results was observed by Dehghani et al. (2016) in subjects with CKD who received two capsules of $500 \mathrm{mg}$ of a synbiotic for six weeks and by Alatriste, Arronte, Espinosa, and Cuevas (2014) in individuals with CKD who received a dose of $16 \times 10^{9} \mathrm{CFU}$ of Lactobacillus casei shirota (LCS) for eight weeks. However, in our study we used a food instead capsule. 


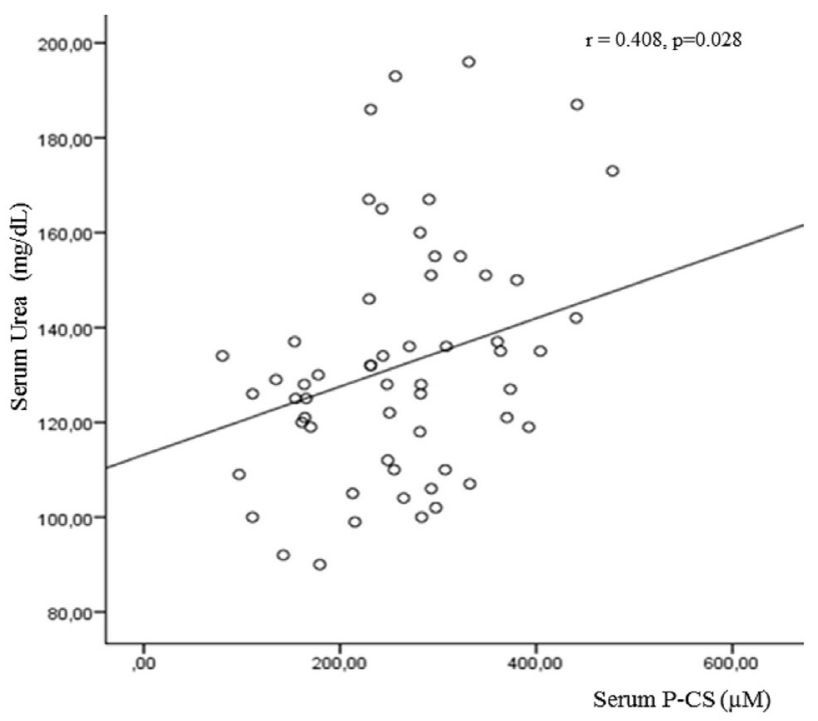

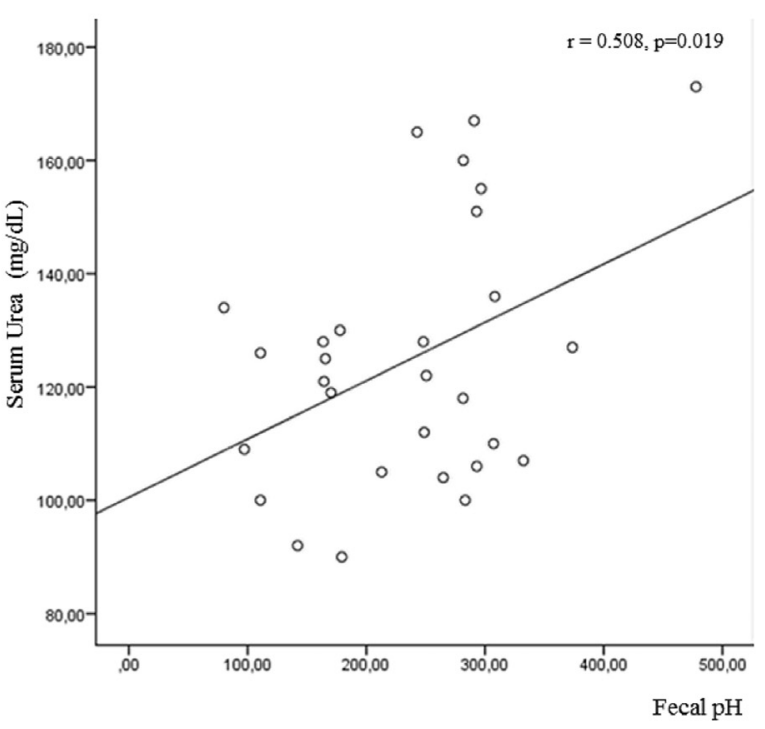

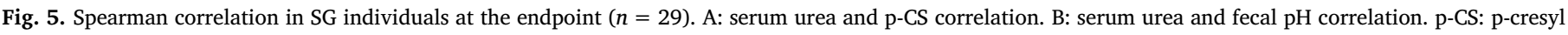
sulfate.

Another important factor is that the decrease of serum uremic toxins values may be related to the lower production of ammonia by the intestinal microbiota, since this is directly related to intestinal permeability (Vaziri, 2016).

Our study also confirmed the acidification of colonic environment, causing fecal $\mathrm{pH}$ reduction and increase in the concentration of acetic, butyric, propionic acids in the HD subjects who received a synbiotic meal. This acidification may be related to ammonia rate, as well as to improve the intestinal microbiota composition and the serum uremic toxins concentration (Borges et al., 2017). The urea concentration was correlated to fecal $\mathrm{pH}$, suggesting the increase of anaerobic bacteria and reduction of uremic toxins. Therefore, urea concentration reduction leads to a decrease in the production of p-CS. Some studies with probiotic have documented the reduction $\mathrm{pH}$ colonic in CKD individuals (Borges et al., 2017; Natarajan et al., 2014). The reduction of serum pCS and IS concentration found in our study may be related to the inhibition of the enzymes involved in generation of p-CS and IS and by increase of fecal butyric acid concentration at the endpoint in the SG. Both cereals are dietary fiber sources because their fiber content is higher than $2.5 \mathrm{~g}$ per serving (ANVISA, 2017). Dietary fibers produce short chain fatty acids when fermented, among them acetic, butyric and propionic acids. However, sorghum present higher concentration of fiber than corn, which caused an increase in the butyric acid concentration and fecal $\mathrm{pH}$ reduction in the synbiotic group (sorghum). This was not observed in the control group (corn). Butyric acid exerts a variety of effects on intestinal function as reinforce the colonic defense barrier through of mucins production and antimicrobial peptides, and decrease intestinal epithelial permeability by increasing the expression of tight junction proteins reducing uremic toxin influx (Tralongo et al., 2014; Vaziri, 2016).

This study also showed a reduction in serum phosphorus concentration in SG at endpoint compared to baseline. In addition, the use of synbiotic meal was able to alter the serum phosphorus and potassium values within the normality values. This result can also be associated with intestinal acidification which can increase the calcium ionization and, consequently, its connection with phosphorus, preventing its absorption (Ogawa et al., 2012).

The present study presented a limitation in relation to protocol control because adherence was not $100 \%$, due to disease outcomes. Although our study was a small single-center study and the number of participants limited us to non-stratification of the products tested, the sample size was adequate to examine the hypotheses. However, further studies should evaluate the isolated effects of the extruded sorghum breakfast meal and unfermented probiotic milk on uremic markers.

\section{Conclusion}

This study suggests that the intake of the synbiotic meal (sorghum extruded breakfast meal plus unfermented probiotic milk) during 7-wk reduced the p-CS and IS serum uremic toxins and urea in HD subjects To prove the beneficial effect of this synbiotic to subjects in HD, future clinical studies that correlate serum uremic toxins and diversity and microbiota fecal are necessary.

\section{Conflict of interest}

The authors declare that they have no conflict of interest.

\section{Acknowledgements}

The authors thank the Foundation for Research Support of Minas Gerais (FAPEMIG, Brazil) (CDS-APQ-01683-15), Coordination for the Improvement of Higher Education Personnel (CAPES, Brazil), National Counsel of Technological and Scientific Development (CNPq, Brazil) and the Embrapa Maize and Sorghum (Brazil) for granting of financial support for undergraduate research and scholarships.

\section{References}

Alatriste, P. V. M., Arronte, R. U., Espinosa, C. O. G., \& Cuevas, M. L. A. E. (2014). Effect of probiotics on human blood urea levels in patients with chronic renal failure. Nutrición Hospitalaria, 29(3), 582-590. https://doi.org/10.3305/NH.2014.29.3.7179. ANVISA (2017). Alimentos Com Alegacoes de Propriedades Funcionais e ou de Saúde.

Ashraf, R., \& Shah, N. P. (2011). Selective and differential enumerations of Lactobacillus delbrueckii subsp. bulgaricus, Streptococcus thermophilus, Lactobacillus acidophilus, Lactobacillus casei and Bifidobacterium spp. in yoghurt-a review. International Journal of Food Microbiology, 149(3), 194-208. https://doi.org/10.1016/j. ijfoodmicro.2011.07.008.

Bansal, N., McCulloch, C. E., Lin, F., Robinson-Cohen, C., Rahman, M., Kusek, J. W., ... Townsend, R. R. (2016). Different components of blood pressure are associated with increased risk of atherosclerotic cardiovascular disease versus heart failure in advanced chronic kidney disease. Kidney International, 90(6), 1348-1356. https://doi. org/10.1016/j.kint.2016.08.009.

Bingham, S. A. G., Welch, C., Cassidy, A., et al. (1994). Epidemiology: Weighed records V. $24 \mathrm{H}$ recalls, food-frequency. British Journal of Nutrition, 72(1994), 619-643. https:// 
doi.org/10.1079/BJN19940064.

Borges, N. A., Carmo, F. L., Stockler-Pinto, M. B., de Brito, J. S., Dolenga, C. J., Ferreira, D. C., ... Mafra, D. (2017). Probiotic supplementation in chronic kidney disease: A double-blind, randomized, placebo-controlled trial. Journal of Renal Nutrition, 1-9. https://doi.org/10.1053/j.jrn.2017.06.010.

Cardoso, L. D. M., Pinheiro, S. S., Martino, H. S. D., \& Pinheiro-Sant'Ana, H. M. (2017). Sorghum (Sorghum bicolor L.): Nutrients, bioactive compounds, and potential impact on human health. Critical Reviews in Food Science and Nutrition, 57(2), 372-390.

Dean, A. G., Sullivan, K. M., \& Soe, M. M (2014). Open source epidemiologic statistics for public health.

Dehghani, H., Heidari, F., Mozaffari-Khosravi, H., Nouri-Majelan, N., \& Dehghani, A. (2016). Synbiotic supplementations for Azotemia in patients with chronic kidney disease: A randomized controlled trial. Iranian Journal of Kidney Diseases, 10 , 351-357.

Evenepoel, P., Meijers, B. K. I., Bammens, B. R. M., \& Verbeke, K. (2009). Uremic toxins originating from colonic microbial metabolism. Kidney International Supplement, 76(114), S12-S19. https://doi.org/10.1038/ki.2009.402.

Fukuuchi, F., Hida, M., Oga, Y., Endoh, M., Kurokawa, K., \& Sakai, H. (2002). Intestinal bacteria-derived putrefactants in chronic renal failure. Clinical and Experimental Nephrology, 6(2), 99-104. https://doi.org/10.1007/s101570200016.

Hill, N. R., Fatoba, S. T., Oke, J. L., Hirst, J. A., O'Callaghan, C. A., Lasserson, D. S., \& Hobbs, F. D. R. (2016). Global prevalence of chronic kidney disease - A systematic review and meta-analysis. PLoS One, 11(7), e0158765.

Ito, S., \& Yoshida, M. (2014). Protein-bound uremic toxins: New culprits of cardiovascular events in chronic kidney disease patients. Toxins, 6(2), 665-678. https://doi.org/10. $3390 /$ toxins6020665.

Jellife, D. B. (1968). Evaluación del estado de nutrición de la comunidad. Ginebra: Organización Mundial de La Salud.

Lipschitz, D. (1994). Screening for nutritional status in the elderly. Primary Care, 21, $55-67$.

Lopes, R. C. S. O., Lima, S. L. L., Silva, B. P., Toledo, R. C. L., Moreira, M. E. C., Anunciação, P., ... Martino, H. S. D. (2018). Evaluation of the health benefits of consumption of extruded tannin sorghum with unfermented probiotic milk in in dividuals with chronic kidney disease. Food Research International, in press.. https:// doi.org/10.1016/j.foodres.2018.03.004.

Meert, N., Schepers, E., Glorieux, G., Van Landschoot, M., Goeman, J. L., Waterloos, M. A., ... Vanholder, R. (2012). Novel method for simultaneous determination of pcresylsulphate and p-cresylglucuronide: Clinical data and pathophysiological implications. Nephrology Dialysis Transplantation, 27(6), 2388-2396. https://doi.org/10 1093/ndt/gfr672.

Moraes, É. A., Marineli, R. D. S., Lenquiste, S. A., Steel, C. J., De Menezes, C. B., Queiroz, V. A. V., \& Maróstica Júnior, M. R. (2015). Sorghum flour fractions: Correlations among polysaccharides, phenolic compounds, antioxidant activity and glycemic index. Food Chemistry, 180(2015), 116-123.

Nakabayashi, I., Nakamura, M., Kawakami, K., Ohta, T., Kato, I., Uchida, K., \& Yoshida, M. (2011). Effects of synbiotic treatment on serum level of p-cresol in haemodialysis patients: A preliminary study. Nephrology, Dialysis, Transplantation, 26(3), 1094-1098. https://doi.org/10.1093/ndt/gfq624.

Natarajan, R., Pechenyak, B., Vyas, U., Ranganathan, P. P., Weinberg, A., Liang, P., ... Saggi, S. J. (2014). Randomized controlled trial of strain-specific probiotic formulation ( Renadyl ) in dialysis patients. BioMed Research International, 2014, 1-9. https://doi.org/10.1155/2014/568571.
Ogawa, T., Shimada, M., Nagano, N., Ito, K., Ando, T., Shimomura, Y., ... Otsuka, K. (2012). Oral administration of Bifidobacterium longum in a gastro-resistant seamless capsule decreases serum phosphate levels in patients receiving haemodialysis. Clinical Kidney Journal, 5(4), 373-374.

Oliveira, D., Vidal, L., Ares, G., Walter, E. H. M., Rosenthal, A., \& Deliza, R. (2017) Sensory, microbiological and physicochemical screening of probiotic cultures for the development of non-fermented probiotic milk. LWT - Food Science and Technology, 79, 234-241. https://doi.org/10.1016/j.lwt.2017.01.020.

Park, S., \& Bae, J.-H. (2015). Probiotics for weight loss: A systematic review and metaanalysis. Nutrition Research, 35(7), 566-575.

Rossi, M., Johnson, D. W., Morrison, M., Pascoe, E. M., Coombes, J. S., Forbes, J. M., ... Campbell, K. L. (2016). Synbiotics easing renal failure by improving gut microbiology (SYNERGY): A randomized trial. Clinical Journal of the American Society of Nephrology, 11(2), 223-231. https://doi.org/10.2215/CJN.05240515.

Rossi, M. M., Kerenaftali, K. K., Johnson, D. W., \& Campbell, K. L. (2012). Pre-, pro-, and synbiotics: Do they have a role in reducing uremic toxins? A systematic review and meta-analysis. International Journal of Nephrology, 2012(1), 1-20. https://doi.org/10. $1155 / 2012 / 673631$.

Siegfried, V. R., Ruckemann, H., \& Stumpf, G. (1984). Method for the determination of organic acid in silage by high performance liquid chromatography. Landwirtsh Forsch, 37.

Skrypnik, K., \& Suliburska, J. (2017). Association between the gut microbiota and mineral metabolism. Journal of the Science of Food and Agriculture(June), https://doi.org/10. 1002/jsfa.8724.

Stockler-Pinto, M. B., Soulage, C. O., Borges, N. A., Cardozo, L. F. M. F., Dolenga, C. J., Nakao, L. S., ... Mafra, D. (2018). From bench to the hemodialysis clinic: Proteinbound uremic toxins modulate NF-kB/Nrf2 expression. International Urology and Nephrology, 50(2), 347-354.

Takayama, F., Taki, K., \& Niwa, T. (2003). Bifidobacterium in gastro-resistant seamless capsule reduces serum levels of indoxyl sulfate in patients on hemodialysis. American Journal of Kidney Diseases, 41(3 Suppl 1), S142-S145. https://doi.org/10.1053/ajkd. 2003.50104.

Tralongo, P., Tomasello, G., Sinagra, E., Damiani, P., Leone, A., Palumbo, V. D., ... Lo Monte, A. I. (2014). The role of butyric acid as a protective agent against inflammatory bowel diseases. EuroMediterranean Biomedical Journal, 9(11), 24-35. https://doi.org/10.3269/1970-5492.2014.9.4.

Vaziri, N. D. (2016). Effect of synbiotic therapy on gut? Derived uremic toxins and the intestinal microbiome in patients with CKD. Clinical Journal of the American Society of Nephrology, 11(2), 199-201. https://doi.org/10.2215/CJN.13631215.

Vaziri, N. D., Zhao, Y. Y., \& Pahl, M. V. (2016). Altered intestinal microbial flora and impaired epithelial barrier structure and function in CKD: The nature, mechanisms, consequences and potential treatment. Nephrology Dialysis Transplantation, 31(5), 737-746. https://doi.org/10.1093/ndt/gfv095.

Vázquez-Araújo, L., Chambers, E., \& Cherdchu, P. (2012). Consumer input for developing human food products made with sorghum grain. Journal of Food Science, 77(10), S384-S389.

WHO.World Health Organization (1997). Obesity: preventing and managing the global epidemic.Report of WHO. Genebra: Consultation on Obesity.

Wong, J., Piceno, Y. M., Desantis, T. Z., Pahl, M., Andersen, G. L., \& Vaziri, N. D. (2014) Expansion of urease- and uricase-containing, indole- and p-cresol-forming and contraction of short-chain fatty acid-producing intestinal microbiota in ESRD. American Journal of Nephrology, 39(3), 230-237. https://doi.org/10.1159/000360010. 